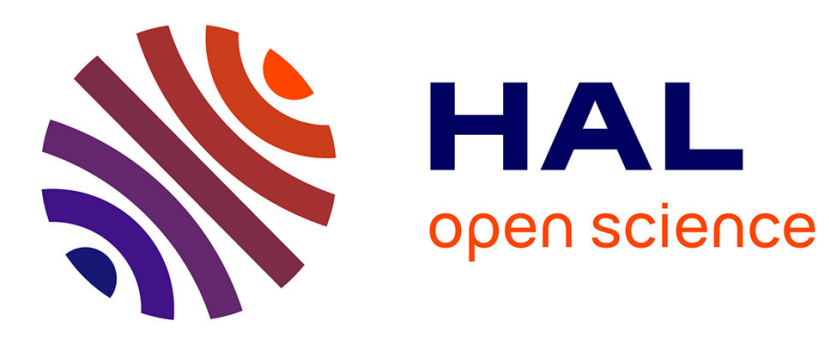

\title{
Classical noise and nonlinear effects in the ideal avalanche diode
}

\author{
A.A. Walma
}

\section{To cite this version:}

A.A. Walma. Classical noise and nonlinear effects in the ideal avalanche diode. Revue de Physique Appliquée, 1980, 15 (11), pp.1585-1597. 10.1051/rphysap:0198000150110158500 . jpa-00244885

\section{HAL Id: jpa-00244885 https://hal.science/jpa-00244885}

Submitted on 1 Jan 1980

HAL is a multi-disciplinary open access archive for the deposit and dissemination of scientific research documents, whether they are published or not. The documents may come from teaching and research institutions in France or abroad, or from public or private research centers.
L'archive ouverte pluridisciplinaire HAL, est destinée au dépôt et à la diffusion de documents scientifiques de niveau recherche, publiés ou non, émanant des établissements d'enseignement et de recherche français ou étrangers, des laboratoires publics ou privés. 


\title{
Classical noise and nonlinear effects in the ideal avalanche diode
}

\author{
A. A. Walma \\ Centre d'Etudes d'Electronique des Solides $\left(^{*}\right)$, Université des Sciences et Techniques du Languedoc, \\ 34060 Montpellier Cedex, France
}

(Reçu le 25 février 1980, révisé le 9 juin 1980, accepté le $1^{\text {er }}$ août 1980)

Résumé. - On présente une formulation générale de la statistique de l'avalanche dans une diode idéale en s'efforçant d'étendre cette théorie au régime non linéaire.

Tous les résultats connus, incluant la dépendance en fréquence, sont décrits par ce procédé. Il unifie les différents traitements de façon consistante et permet une analyse de phénomènes non linéaires. Quand le régime transitoire de l'injection et les différentes vitesses de dérive deviennent importants, la Invariant Imbedding Technique est appliquée.

Les limitations des équations de continuité qui constituent le point de départ du traitement statistique sont discutées. On a donné un exemple où les méthodes classiques (Langevin, théorème de G.-R., petits signaux, analyse de Fourier) ne sont plus applicables dans le cas non linéaire.

\begin{abstract}
A general formulation of the avalanche statistics in an ideal diode is presented, in an effort to extend the theory to the nonlinear regime. All known results, including the time dependency, are recovered with this procedure. It unifies the various treatments in a consistent way and enables an analysis of nonlinear phenomena. Where the injection transient and different drift velocities become important, the invariant imbedding technique is used. The limitations of the continuity equations as a starting point for a statistical treatment are discussed. An example is given where the classical methods (Langevin, G.-R. theorem, a-c perturbations, Fourier analysis) fail in the nonlinear case.
\end{abstract}

\section{List of symbols}

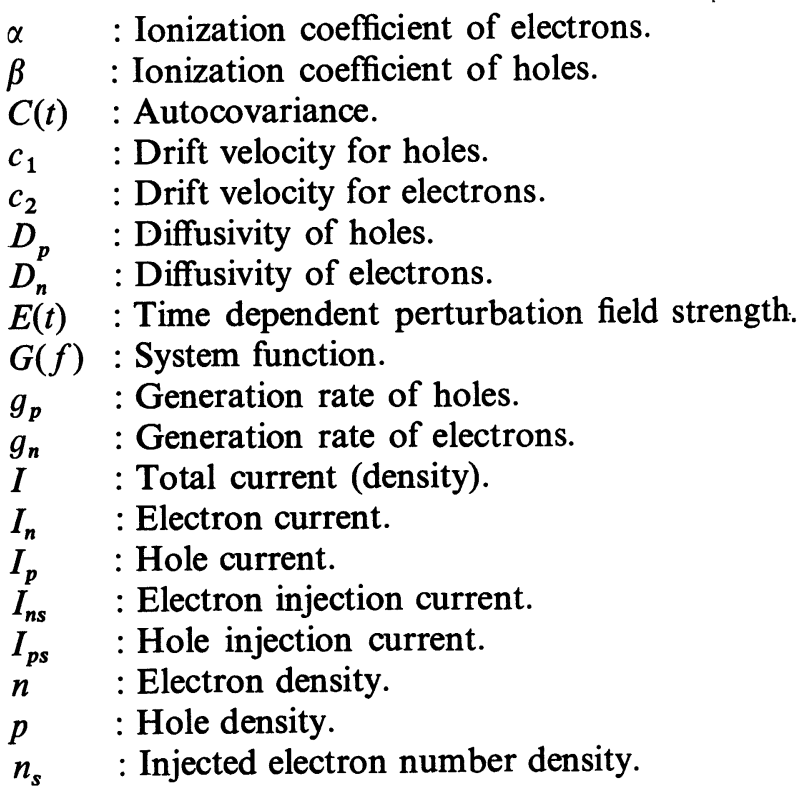

(*) Associé au C.N.R.S. $n_{p} \quad:$ Injected hole number density.

$N \quad$ : Total number of pairs in the active region.

$N_{s} \quad$ : Total injected pairs.

$N_{s n} \quad$ : Total injected electron number.

$N_{s p} \quad$ : Total injected hole number.

$M \quad$ : Multiplication factor for equal ionization coefficients.

$M_{n} \quad$ : Multiplication factor for electrons for unequal ionization coefficients.

$M_{p} \quad$ : Multiplication factor for holes for unequal ionization coefficients.

$n_{0} \quad$ : Deterministic number density of pairs in the active region.

$p_{n}(t)$ : Probability of $n$ entities at a certain time $t$.

$P \quad$ : Generating function of some fluctuating parameter.

$Q_{\mathrm{L}} \quad$ : Generating function of the number of pairs in the active region caused by the leftgoing particle of a pair created at an arbitrary position within the region.

$Q_{\mathrm{R}} \quad$ : Generating function of the number caused by the rightgoing particle.

$Q_{\mathrm{P}} \quad$ : Generating function of the pairs. 
$q \quad$ : Small parameter characterizing the nonlinearity of the continuity equation for pairs.

$R(t)$ : Number of particles up to time $t$ returning from an active region after a single injection.

$r_{e} \quad$ : Rate of returning electrons.

$r_{p} \quad$ : Rate of returning holes.

$s \quad$ : Arbitrary parameter smaller than one.

$S_{N}(f)$ : Spectral density of total number of pairs.

$S_{I}(f)$ : Current spectral density.

$\tau_{\mathrm{in} n} \quad:$ Intrinsic response time for electrons.

$\tau_{\mathrm{i} p} \quad:$ Intrinsic response time for holes.

$\tau_{t} \quad:$ Transittime.

$\tau_{p} \quad$ : Recombination life time of holes.

$\tau_{n} \quad$ : Recombination life time of electrons.

$\lambda_{n} \quad$ : Functional rate of increase for electrons.

$\mu_{n} \quad$ : Functional rate of decrease for electrons.

$\gamma(n)$ : Nonlinear functional rate of increase for pairs.

1. Introduction. - An attempt is made to present a unified approach to the various noise treatments of the ideal avalanche diode. This is done by analysing the kinetic equations of the active region by means of a general equation of Markov Processes [1, 2]. Classically, random sources and perturbing fields are used for the calculation of the noise and time dependent properties. In this paper we start with a characteristic function or generating function, from which it is known that it describes the stochastic process under study completely $[1,2]$. No random sources or a-c excitations are needed this way. For linear processes, or in those cases where it can be linearized, the usual treatments (G.-R. theorem, Burgess' method, simple Langevin, perturbative fields, etc.) are equivalent to the construction of a diffusion limit of the process under concern. It implies that the actual physical phenomenon is described by a socalled Ornstein-Uhlenbeck process [3]. This process needs a sharply peaked distribution at the equilibrium value however, in order to be able to linearize. We shall avoid these limitations and start directly with the Chapman-Kolmogorov (C.-K.) equation [4] for the probability density function in terms of a characteristic function. This puts the noise treatment probabilistically on a much firmer basis since the use of the C.-K. equation actually implies that we start our analysis with the total probability theorem [4]. Some new results are obtained and others are modified slightly. The proposed procedure is also valid in the nonlinear case, which cannot always be said of the diffusion limit [5].

As far as the first order properties are concerned, our starting point will be in the first place the simple Read equation [6] which is valid for equal ionization coefficients and drift velocities. In order to appreciate its stationary or equilibrium character in a better way, this equation will be rederived in section 2 . For unequal ionization coefficients and drift velocities, the results from [7] are used. In this contribution it was shown that the ratio of the hole and electron ionization coefficients had an important effect on the bandwidth obtained from an avalanche photodiode. An intrinsic response time was introduced. On a macroscopic scale the derived continuity equations may be accurate, but unfortunately some statistical information has been lost. This will be further discussed in section 4. For this case some results of the invariant imbedding technique will be used [8].

As for the second order properties, the noise behaviour of the avalanche photodiode is well established by now and its qualitative features verified experimentally in a satisfactory way. This does not mean that everything is clear yet on a microscopic scale. Often the ratio of the ionization coefficients is taken to be independent of the field strength. This approximation is certainly not a good one for all semiconductors (it is even a poor approximation for $\mathrm{Si}$. Nevertheless the qualitative features of the various noise theories are generally accepted. In the nonlinear case (space charge effects for instance) we are still in a poor state. It has not been possible yet to solve rigorously the simplest (inherently) nonlinear problem in a statistical sense. It may be worthwhile to point out once more that the procedures based on a diffusion limit (Langevin, G.-R., etc.) may not be accurate for this case. As for the photodiode three different approaches have been used resulting together into a general expression for a non-white spectral density of the multiplied current.

The first explicit results were obtained in [9]. For the time dependency Read's equation was used and the variance of the ionizing process was shown to be proportional to the third power of the multiplication factor, whereas the cut-off frequency depended on the first power of the multiplication factor. This cubic relationship and multiplied transittime are the essential ideas underlying all subsequent theories. These results were derived along different lines. We will show that they follow quite naturally from the C.-K. equation.

In [10] this analysis was extended to the case where the ionization coefficients for electrons (denoted by $\alpha$ ) and for holes (denoted by $\beta$ ) were unequal. A stationary variance was derived for both the electron and hole current (note that both expressions are symmetric in $\alpha$ and $\beta$ ). This was done by considering a small increment of the active region and studying its shot noise contribution to the noise spectral density of the whole layer. It is therefore worked with a Langevintype of approach where the random source is supposed to be of the shot type. Also for this case, the obtained results follow quite naturally from the C.-K. equation of the multiplication factor. This is of importance if the analysis is extended to the nonlinear case, since the Langevin procedure and shot noise assumption imply that no correlations exist between the increments of the active region so that the classical 
treatments (diffusion limit of a stochastic process) may fail.

The time dependent properties for unequal $\alpha$ and $\beta$ were discussed in [11, 12]. Though the attenuation of the noise current at high frequencies was already mentioned in [10] and explicitly worked out in [9] for the Read case, these contributions give the complete result (including external generation within the active region [11]) in terms of the intrinsic response time [7]. To that purpose the time dependent multiplication factor, in the presence of a small perturbing field, was introduced in McIntyre's expression for the stationary noise. Again, this macroscopic attack treats the process in the diffusion limit and may not be valid in the nonlinear case. We shall derive similar results by using the invariant imbedding technique [8] and show their limitation in the high frequency limit. The obtained time constant differs from the intrinsic response time. The invariant imbedding technique includes the transient after injection and yields expressions for the rate of emergence from the active region at every time $t$.

For those applications where the performance bound of the diode becomes important (PCM systems for instance) one may wish to know the probability that a given pulse of photons, generating a number of photo carriers, will be detected or not. We are therefore interested in the probability density function of the number of pairs in the active region after injection of a certain number of electrons or holes. The first explicit result was obtained in [13]. The same problem was dealt with in [14] on the basis of a probability transform for the number of pairs in the active region. The final result was an implicit equation for the moment generating function. It contains the results from [10] and [13] which were not derived explicitly at that time however. Both approaches are rather different. We will show that the central result from [14] is the backward version of a birth process with parameter $\alpha-\beta$. This is done either by means of the continuity equation for the multiplication factor or by means of an internal source discussed in [8]. In both cases the C.-K. equations are used.

The general handicap of the obtained results in avalanche noise literature is, that it is started with continuity equations in equilibrium. Especially for largely differing drift velocities, the transient after injection can cause deviations not only in the high frequency limit but also for intermediate frequencies, thus influencing the gain bandwidth product. This does not seem to be desastrous in practice since the qualitative features of the multiplied intrinsic response time from [7] could be more or less confirmed experimentally. More details will be given in section 5 .

A qualitative discussion about the statistical treatment of the ideal avalanche diode including nonlinear effects such as space charge is presented in section 6. A simple model is proposed in order to show the inadequacy of the classical noise treatments. It is hoped to deal with this problem elsewhere in more detail by using the general equation for Markov processes.

We finally list the conditions which are imposed upon the diode :

(i) A uniform breakdown and field pattern are assumed ;

(ii) The effects of a displacement current are negligible ;

(iii) Constant ionization coefficients are assumed, which may be different but are functions of the electric field only;

(iv) We deal with a single type of injection (for convenience) and uniform drift velocities without internal generation ;

(v) No space charge effects are allowed.

Experimental evidence shows that this highly idealized behaviour is not too unrealistic on several occasions. As will be shown in the sequel, even for this idealized case it is sometimes difficult to find explicit results.

2. Theory. - Whether we apply the invariant Imbedding technique [8] or the general equation for Markov processes [5], at the basis one always finds the Chapman-Kolmogorov relation [4], the validity of which can be proven by the theorem of total probability. For details about a Markov process we like to refer to [3] and [4]. It is assumed, that with a stationary input, the multiplication process will be ergodic, which means that the limiting probability of a certain number of created pairs exists, irrespective of the initial distribution.

The starting point is a Markov process with a finite or denumerably infinite number of states. Our statespace consists of the number of created pairs plus input. The path from the transition probabilities, determined by the physics of the problem, to Chapman-Kolmogorov's differential equations has become standard by now and can be found in many books on the theory of stochastic processes [1-4]. For a Markov process with local changes (Birth and Death process/Master-equation) the forward version can be written as $[3,4]$ :

$$
\begin{aligned}
\frac{\partial p_{n}}{\partial t}=g(n-1) p_{n-1}+r(n+1) p_{n+1} & - \\
& -(g(n)+r(n)) p_{n} .
\end{aligned}
$$

The rates $r(n)$ and $g(n)$ describe the transitions of one up or one down and $p_{n}$ is the probability that the random variable under study has a certain value $n$ at a certain time $t$. On many occasions it is easier to work with a probability transform. We choose a simple generating function $[1,3]$ :

$$
P(s, t)=\sum_{k=0}^{\infty} p_{k}(t) s^{k}, \quad|s| \leqslant 1
$$


This function can be defined on any converging sequence of real numbers and is of central importance in the handling of stochastic processes involving integral valued random variables. There are of course a number of other functions that generate moments and cumulants (moment generating functions, characteristic functions, etc.). For our discrete state space the simple generating function (GF) will be used.

On a macroscopic scale, the processes under consideration are linear so that the evaluation of the random variable with respect to time can be written as

$$
\frac{\mathrm{d} n}{\mathrm{~d} t}=g(n)-r(n)
$$

The transition rates are thus linear in the state variables. Multiplying (1) with $s^{k}$ and summing over $k$ yields then :

$$
\begin{aligned}
\frac{\partial P}{\partial t}=s g\left(s \frac{\partial}{\partial s}\right) P+ & s^{-1} r\left(s \frac{\partial}{\partial s}\right) P- \\
- & {\left[g\left(s \frac{\partial}{\partial s}\right)+r\left(s \frac{\partial}{\partial s}\right)\right] P }
\end{aligned}
$$

where the differential operator acts upon $P$ only.

Rearranging (4) :

$$
\frac{\partial P}{\partial t}=(s-1) g\left(s \frac{\partial}{\partial s}\right) P+\left(s^{-1}-1\right) r\left(s \frac{\partial}{\partial s}\right) P .
$$

Representing the transition rates by a single function, the general equation for Markov processes from $[1,2]$ is found in terms of a GF. This is our central result, which will be used for the continuity equations of the avalanche region. It contains the G.-R. theorem and Burgess' method as special cases and can also be applied to the nonlinear case [5].

For the spectrum Burgess' method could be employed [15]. For rates nonlinear in their state variables however, this diffusion limit should be handled with care [5]. We shall not go into this matter since the usual continuity equations of the avalanche region are linear. For the density functions of the number of pairs, equation (5) will be used. With the help of this equation the major bulk of the noise results from literature can be recovered.

Consider for instance the central result from [14] equation (7). It represents an implicit equation for the moment generating function of the number of created pairs. In [16] Lagrange's formula was used for its reversion. The obtained distribution function coincided with the one derived in [13]. Its variance yields the results from [9] and [10]. All these expressions for the stationary case were obtained along different lines and sometimes quite elaborate. We therefore like to show how the analysis can greatly be simplified by using equation (5). It was suggested in [8 II] that this expression for the moment generating function represented the backward version of a simple birth process. In order to show this, consider the mean of the pairs caused by an internal generation [8 III eq. (5)] at $y$ somewhere in the active region :

$$
\frac{\mathrm{d} \bar{N}_{y}}{\mathrm{~d} y}=(\beta-\alpha) \bar{N}_{y} .
$$

For an injected carrier the same equation holds but with a different boundary condition. Denoting the number of created pairs (which is equivalent with the multiplication) by the arbitrary variable $n$ we then study the process

$$
\frac{\mathrm{d} n}{\mathrm{~d} y}=(\beta-\alpha) n
$$

where $n$ is now a random variable. This represents a simple birth process, the solution of which can be found in many books on the theory of branching processes, stochastic processes, queueing theory, etc. The application of equation (5) leads to the following partial differential equation for the GF (forward version) :

$$
\frac{\partial P}{\partial x}=(\beta-\alpha)(s-1) s \frac{\partial P}{\partial s}
$$

where the running variable is now the distance instead of the time. Following [1] $\mathrm{Ch}$. 3, for the backward version, the GF of the transitions occurring in $\Delta x$ can be written as :

$$
P(s, \Delta x)=s+g(s) \Delta x+0(\Delta x) .
$$

For multiplicative processes, such as are given by equation (6), the following standard result exists $[1,3,4]$ :

$$
P(s, x+\Delta x)=P[P(s, x), \Delta x] .
$$

Expanding (9) around $x$, after substitution of (8), yields

$$
\frac{\partial P}{\partial x}=g[P] .
$$

In order to find the function $g(s)$, it is noted that on the basis of (6) :

$$
\begin{aligned}
& P(s, x)=p_{0}+p_{1} s+p_{2} s^{2}+\cdots= \\
& =s[1-(\beta-\alpha) \Delta x]+(\beta-\alpha) \Delta x s^{2}+0(\Delta x)^{2}
\end{aligned}
$$

since $p_{0}=0, p_{1}=1-(\beta-\alpha) \Delta x$ and $p_{2}=(\beta-\alpha) \Delta x$ with all other possibilities of the order of $(\Delta x)^{2}$. This yields for the required function

$$
g(s)=(\beta-\alpha) s(s-1) .
$$

Combining (11) with (10) :

$$
\frac{\partial P}{\partial x}=(\beta-\alpha) P(P-1) .
$$


This is exactly equation (7) from [14] containing the necessary information for the statistical results of $[10,13]$.

This important equation can also be obtained from the stochastic invariant imbedding technique [8 III]. The fluctuating properties of the number of emerging particles are studied by means of an internal source. The creation of an extra electron-hole pair at an arbitrary point $y$ within the active region, causes an outcoming particle stream due to the leftgoing and rightgoing carrier at $y$. Two GF's were defined satisfying the following set of equations :

$$
\begin{aligned}
& \frac{\partial Q_{\mathrm{L}}}{\partial y}=-\alpha Q_{\mathrm{R}} Q_{\mathrm{L}}^{2}+\alpha Q_{\mathrm{L}} \\
& \frac{\partial Q_{\mathrm{R}}}{\partial y}=\beta Q_{\mathrm{R}}^{2} Q_{\mathrm{L}}-\beta Q_{\mathrm{R}}
\end{aligned}
$$

where $Q_{\mathrm{L}}$ and $Q_{\mathrm{R}}$ represent the GF's for the number of particles coming out of the avalanche region that are caused by the leftgoing and the rightgoing carrier at $y$ respectively. Using the general property of GF's that the sum of two independent random processes can be characterized by the product of the respective GF's [3, 4], we get for the pairs :

$$
Q_{\mathrm{P}}=Q_{\mathrm{L}} Q_{\mathrm{R}}
$$

from which

$$
\frac{\partial Q_{\mathrm{P}}}{\partial x}=Q_{\mathrm{L}} \frac{\partial Q_{\mathrm{R}}}{\partial x}+Q_{\mathrm{R}} \frac{\partial Q_{\mathrm{L}}}{\partial x} .
$$

Together with (13)

$$
\frac{\partial Q_{\mathbf{P}}}{\partial x}=(\beta-\alpha) Q_{\mathbf{P}}\left(Q_{\mathbf{P}}-1\right) .
$$

Identifying $Q_{\mathrm{P}}$ with $P$ from (12), it follows that the central result for the time independent statistics also follows from the invariant imbedding technique.

Summarizing, for the stationary fluctuations of the avalanche process in an ideal diode [9, 10, 13, 14], the multiplication statistics can be described by the backward version of a birth process with $\beta-\alpha$ as a birth rate :

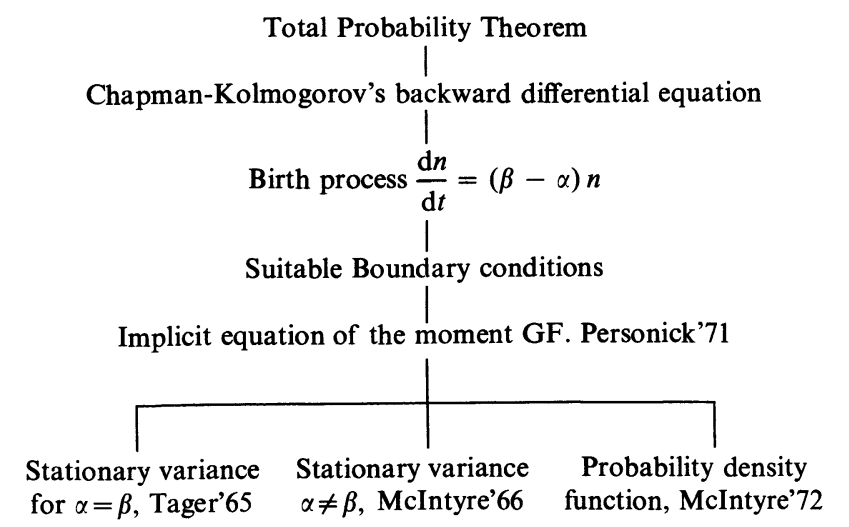

Including the time dependency into these calculations is more difficult than it may seem at first sight. It was already suggested and verified in [17] that the ratio of the ionization coefficients and the multiplication factor had an important effect on the bandwidth of the diode. This led to the suggestion of a frequency dependent multiplication and the introduction of a socalled intrinsic response time $[7,11$, 12]. In general, one studies the macroscopic behaviour under influence of an a-c perturbation and considers the frequency dependence of the multiplication factor $[12,13]$. Two complications arise with this kind of treatment. (i) It only holds in the linear case. For nonlinear problems, the joint density at separate times should be calculated explicitly. This is possible, in principle, with equation (5). (ii) The starting point are continuity equations that only are defined in a steady state. Non-equilibrium cases can be treated but information about the transient is lost. This can play an important role for unequal drift velocities. To handle this, the invariant imbedding technique will be used. For a better understanding of their equilibrium and steady state character, the basic rate equations and the simple Read equation will be rederived [18].

2.1 RATE eQuation. - In any semiconducting crystal electron and hole pairs are continually being generated by thermal or other means and are continually recombining. The continuity equations describing these effects are :

$$
\begin{aligned}
& \frac{\partial p}{\partial t}=-\nabla I_{p}+g_{p}-\frac{p}{\tau_{p}} \\
& \frac{\partial n}{\partial t}=-\nabla I_{n}+g_{n}-\frac{n}{\tau_{n}}
\end{aligned}
$$

$I_{p}$ and $I_{n}$ denote the particle flux densities of holes and electrons, $g_{n}$ and $g_{p}$ the actual generation rates of electrons and holes respectively and $\tau_{p}$ or $\tau_{n}$ the mean carrier lifetime. As far as a stochastic treatment is concerned, we are interested in the local concentrations of $n$ and $p$. The solutions of (14), under appropriate boundary conditions, describe the distribution of electron and hole concentration as a function of space coordinates and time and give a description of the behaviour of the carriers in the semiconductor under equilibrium and non-equilibrium conditions. For our purposes $I_{p}$ and $I_{n}$ are expressed into $p$ and $n$. Since the particle flux density is the sum of a diffusion flux density and a drift current density (from any electric field) :

$$
\begin{aligned}
& I_{p}=-D_{p} \nabla p+p \mu_{p} E, \\
& I_{n}=-D_{n} \nabla n-n \mu_{n} E .
\end{aligned}
$$

Under avalanching conditions the diffusivities $D_{p}$ and $D_{n}$ are assumed to be practically nonexisting. Substitution of (14) in (15) and (16) and noting 
that $\mu_{p} E=c_{1}$ and $\mu_{n} E=c_{2}$, which represent the drift velocities for holes and electrons respectively, results in the one dimensional case into :

$$
\begin{aligned}
& \frac{\partial p}{\partial t}=-c_{1} \frac{\partial p}{\partial x}+g_{p}-\frac{p}{\tau_{p}} \\
& \frac{\partial n}{\partial t}=c_{2} \frac{\partial n}{\partial x}+g_{n}-\frac{n}{\tau_{n}} .
\end{aligned}
$$

The field $E$ in (15) and (16) is the sum of the applied field and the internal field caused by the charged carriers. For the time being this internal influence is neglected and $E$ is simply identified with the externally applied field which is assumed to be independent and of a constant value within the active region, with $c_{1}$ and $c_{2}$ being the saturated drift velocities.

With a typical transittime of the order of $10^{-12}$ it will be clear that the influence of the relatively slow processes governing the recombination of carriers can be neglected. The actual generation rates are in our case determined by impact ionization and therefore proportional to the respective particle densities. Our starting point is then the following set of equations :

$$
\begin{aligned}
& \frac{1}{c_{1}} \frac{\partial p}{\partial t}+\frac{\partial p}{\partial x}=\alpha n+\beta p \\
& \frac{1}{c_{2}} \frac{\partial n}{\partial t}-\frac{\partial n}{\partial x}=\alpha n+\beta p .
\end{aligned}
$$

For the time being our considerations are limited to the case where $\alpha=\beta$. It is then possible to combine (19) and (20) into a single expression. For equal ionization coefficients the sum of the particle densities $n$ and $p$ is constant throughout the region and denoted by $N$. Because of this, (19) and (20) can be integrated with respect to the region width $x$ :

$$
\tau_{t} \frac{\partial N}{\partial t}=-\left.(p-n)\right|_{0} ^{x}+2 \alpha x N
$$

with $\tau_{t}$ representing the transittime. The appropriate boundary conditions are :

$$
\begin{array}{ll}
x=0, & p-n=2 p_{s}-N \\
x=x, & p-n=-2 n_{s}+N
\end{array}
$$

since $p+n=N$. After substitution :

$$
\frac{\tau_{t}}{2} \frac{\partial N}{\partial t}=N_{s}+N[\alpha x-1]
$$

This is the wellknown Read equation. It furnishes a complete description of the stochastic behaviour of the number of pairs.

The use of this equation therefore imposes the following extra conditions (additional to those already mentioned in the introduction for the ideal diode) :

(i) Short transittimes, in order to be able to neglect recombination effects. (ii) The internal field caused by the charged carriers is neglected.

(iii) The generation by impact ionization is dominating.

Point (ii) has been included into the time dependent analysis of [19]. It does not seem to affect the general nature of the statistics at first sight, since only the absolute values of the multiplication and time constant are modified. We shall not deal with its effects any further at this stage.

3. Statistics of the Read equation $\left(\alpha=\beta, c_{1}=c_{2}\right)$. - Consider the evaluation of the number of pairs in the active region with respect to time as is given by equation (21) :

$$
\frac{\tau_{t}}{2} \frac{\partial N}{\partial t}=N_{s}+\alpha x N-N .
$$

This equation was derived long before the influence of the multiplication process on the bandwidth of the avalanche diode became evident. On the basis of equation (5) however, the statistics and the time dependency are completely determined :

$\frac{\tau_{t}}{2} \frac{\partial P}{\partial t}=N_{s}(s-1) P+\alpha x(s-1) s \frac{\partial P}{\partial s}+(1-s) \frac{\partial P}{\partial s}$.

The variance in equilibrium can be obtained from

$$
N_{s} P+(\alpha x s-1) \frac{\partial P}{\partial s}=0 .
$$

Solving (23) shows that the GF can be written as :

$$
P=\left[\frac{1-\alpha x}{1-\alpha x s}\right]^{N_{s} / \alpha x} .
$$

which yields for the equilibrium density :

$$
p_{N}(\infty)=\left(\begin{array}{c}
N_{s} / \alpha x+N-1 \\
N
\end{array}\right)(1-\alpha x)^{N_{s} / \alpha x}(\alpha x)^{N} .
$$

The probability that with a constant injection the avalanche dies out is given by

$$
p_{0}=[1-\alpha x]^{N_{s} / \alpha x}
$$

Or in terms of the multiplication factor $M$ for equal ionization coefficients :

$$
M=\frac{1}{1-\alpha x}
$$

the extinction probability can be written as :

$$
p_{0}=M^{-M N_{s} / M-1} \approx M^{-N_{s}}, M \gg 1 .
$$


This result could be used as a measure for the turnoff probability of a microplasma.

As for the spectral density of the pairs in the active region, it can be seen from (21) that the time constant $\tau$ governing the relaxation back towards the equilibrium value

is equal to

$$
\bar{N}=M N_{s}
$$

$$
\tau=M \tau_{t} / 2 .
$$

In this linear case the autocovariance follows from the macroscopic equation. The regression of $\Delta N(t)$ upon its initial value has an exponential law :

$$
\begin{gathered}
\frac{\tau_{t}}{2} \frac{\partial \Delta N}{\partial t}=\alpha x \Delta N-\Delta N=-\frac{\Delta N}{M} \\
\Delta N(t)=\Delta N(0) \mathrm{e}^{-t / \tau}, \tau=M \frac{\tau_{t}}{2} .
\end{gathered}
$$

Multiplying (32) with $\Delta N(0)$ and averaging yields for the autocovariance

$$
C(t)=\overline{\Delta N(t) \Delta N(0)}=\overline{\Delta N^{2}(0) \mathrm{e}^{-t / \tau}} .
$$

The spectral density is now obtained from the Wiener-Khinchine theorem :

$$
S_{N}(f)=4 \overline{\Delta N^{2}(0)} \frac{\tau}{\omega^{2} \tau^{2}+1}
$$

The variance $\overline{\Delta N^{2}(0)}=\overline{\Delta N^{2}}$ can be obtained from (24) since

$$
\left.\frac{\mathrm{d}^{2} P}{\mathrm{~d} s^{2}}\right|_{s=1}=\overline{N(N-1)}
$$

which can be seen from the definition of the GF :

$$
P(s, t)=\sum_{k=0}^{\infty} p_{N}(t) s^{k} .
$$

In other words :

$$
\overline{\Delta N^{2}}=\left.\frac{\mathrm{d}^{2} P}{\mathrm{~d} s^{2}}\right|_{s=1}+\left.\frac{\mathrm{d} P}{\mathrm{~d} s}\right|_{s=1}-\left[\left.\frac{\mathrm{d} P}{\mathrm{~d} s}\right|_{s=1}\right]^{2} .
$$

Applying this to (24) :

$$
\overline{\Delta N^{2}}=M^{2} N_{s} \frac{\tau_{t}}{2} .
$$

The number spectral density on the basis of equation (21) now follows from (34), (38) and (30) :

$$
S_{N}(f)=4 M^{3} N_{s} \frac{\left(\tau_{t} / 2\right)^{2}}{\omega^{2} M^{2}\left(\frac{\tau_{t}}{2}\right)^{2}+1} .
$$

The cubic relationship with respect to the multiplication factor has already become evident.
Note : It would have been easier to use Burgess' method [15] for the determination of the variance and the time constant of the process described by equation (21). This is not done here, since it obscures the statistical origins somewhat. The point we want to make is, that starting with equation (5) all statistical results follow quite naturally from Chapman-Kolmogorov's equations, which are rigorously established in the theory of stochastic processes. One can then avoid such ingeneous tricks as a random Langevin source $[7,9,11,12]$, a-c excitations $[7,11,12]$ and subsequent Fourier analyses, and maximizod probabilities (Burgess). These methods need sharply peaked equilibrium densities, large populations and the central limit theorem. They are quite convenient in the linear case or on those occasions where it can be linearized. Transitions that are nonlinear and of the same order in their state variables may not belong to this class of processes. These linear treatments may therefore become inaccurate in the nonlinear case [5], that is, if space charge effects are to be included for instance.

The problem is now to convert the number spectral density into a current spectral density. A single injected carrier causes a current $e / \tau_{t}$, where $e$ is the electronic charge and $\tau_{t}$ the transittime. If a pair is created at point $x^{\prime}$ in the active region, the time integral, over the whole region for the electron and hole together, yields again the electronic charge sothat we write for the total current :

$$
I=N \frac{e}{\tau_{t}} .
$$

Another way is by considering the shot noise character of introducing electrons or holes into the system (= active region). It may be worthwhile to note at this stage that if this is not governed by a process with independent increments (e.g. shot-type of arrival), the whole analysis has to be reconsidered. For the time being we assume independent events however. The increase of pairs is determined (see equation (21)) by

$$
\frac{2}{\tau_{t}}\left[N_{s}+\alpha x \bar{N}\right] .
$$

Using (27) and (29) and noting that the current can be written as $\left(40^{\prime}\right)$ which is then multiplied by the electronic charge :

$I=\frac{2 e}{\tau_{t}}\left[N_{s}+\frac{M-1}{M} M N_{s}\right]=2 M N_{s} \frac{e}{\tau_{t}}=2 \bar{N} \frac{e}{\tau_{t}}$.

This way of establishing a relation between number density and current shows a difference of a factor two. This will be due to the steady state character of the Read equation. The pairs are introduced into the system on the average at half the transit. The factor $N$ of (40) becomes $2 \mathrm{~N}$. For the spectral densities it follows :

$$
S_{I}(f)=4\left(\frac{e}{\tau_{t}}\right)^{2} S_{N}(f) .
$$


Together with (39) and (42) we finally obtain for the current spectral density on the basis of Read's equation and from stochastic first principles :

$$
S_{I}(f)=\frac{2 e J_{\mathrm{m}} M^{3}}{\omega^{2} M^{2}\left(\frac{\tau_{t}}{2}\right)^{2}+1}
$$

where $J_{\mathrm{m}}=2 N_{s} e$. This result agrees with those from $[9,10,11]$ which were obtained by linear procedures that may not be valid in the nonlinear case.

As a final remark, the conversion number spectral density to current spectral density can also be obtained by means of the usual definition for the current density :

$$
j=n e v .
$$

Since $v=x / \tau_{t}$, where $x$ represents the active region length, the total current can be written as :

$$
I=j A=n e A \frac{1}{\tau_{t}}
$$

with $A$ being the effective cross-section and $n$ the number density. For the total number $N$, the total current becomes :

$$
I=\frac{N e A}{A x} \frac{1}{\tau_{t}}=N \frac{e}{\tau_{t}}
$$

which agrees with (40).

3.1 Reflection statistics $\left(\alpha=\beta, c_{1} \neq c_{2}\right)$. The conditions imposed upon the Read equation enable an approximate equation for the number of emerging particles from an avalanche region on the basis of the invariant imbedding technique for different drift velocities ( $c_{1}$ for holes and $c_{2}$ for electrons) after injection of an hole or an electron. This treatment describes the avalanche more completely than the usual continuity equations such as those given by (19) and (20). It includes the transient and gives explicit results for unequal drift velocities.

The rate of emergence of the total number of carriers up to time $t$ from the avalanche region can be represented by $[8 \mathrm{I}]$

$$
\begin{aligned}
\frac{\partial r}{\partial x}+\frac{1}{c_{p}} \frac{\partial r}{\partial t}=(\alpha+\beta) r+\alpha c_{1} & \int_{0}^{t} r(x, \tau) \times \\
& \times r(x, t-\tau) \mathrm{d} \tau
\end{aligned}
$$

with $r(0, x)=\frac{\beta c_{1}}{c_{1}+c_{2}}, r(t, 0)=0$ and $c_{p}=\frac{c_{1} c_{2}}{c_{1}+c_{2}}$.

The following situation is envisaged :

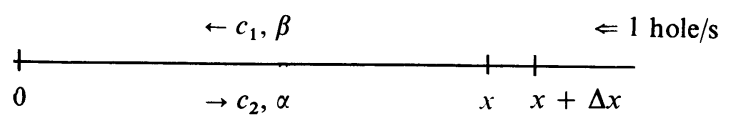

Note that for equilibrium $(t \rightarrow \infty)$ equation (12) from [8 II] is recovered.

For small $t$ equation (40) yields the following set of equations for hole and electron injection respectively

$$
\begin{array}{r}
\frac{\partial r_{e}}{\partial x}+\frac{1}{c_{p}} \frac{\partial r_{e}}{\partial t}=(\alpha+\beta) r_{e}, \\
-\frac{\partial r_{p}}{\partial x}+\frac{1}{c_{p}} \frac{\partial r_{p}}{\partial t}=(\alpha+\beta) r_{p} .
\end{array}
$$

Writing for $r_{e}+r_{p}=R$ it can be integrated over the avalanche region while using the boundary conditions

$$
\begin{array}{ll}
x=0, & r_{e}-r_{p}=2 N_{s n}-R \\
x=x, & r_{e}-r_{p}=R-2 N_{s p} .
\end{array}
$$

As was stated before we take for convenience a single type of injection (electrons). Summing (45) and (46) then yields :

$$
\frac{x}{c_{p}} \frac{\partial R}{\partial t}=2 N_{s n}+(\alpha+\beta) x R-2 R .
$$

For equal ionization coefficients :

$$
\frac{x}{2 c_{p}} \frac{\partial R}{\partial t}=N_{s n}+\alpha x R-R
$$

For equal drift velocities the Read equation is recovered, but now with twice the time constant. It shows its steady state character. The input current from (43) now becomes $N_{s n} e$, which is more realistic since $N_{s n}$ represents electron injection. The initial stages of an avalanche for equal ionization coefficients are thus well described by the Read equation. Equation (47) represents its extension to unequal drift velocities.

4. Statistics of the continuity equations for $\alpha \neq \beta$ and $c_{1} \neq c_{2}$ - - In [7] the time dependent continuity equations for electrons and holes (eqs. (19) and (20)) were studied and the following differential equation was obtained for the total particle current :

$$
\frac{\partial J}{\partial t}=-\frac{J}{M_{n} \tau_{\mathrm{in}}}+\frac{J_{s n}}{\tau_{\mathrm{in}}}
$$

where we have neglected the hole injection. $M_{n}$ appears to be the multiplication factor for electrons

$$
M_{n}=\frac{\beta-\alpha}{\beta-\alpha e^{(\beta-\alpha) x}} .
$$

The intrinsic response time $\tau_{\text {in }}$ can be written as :

$$
\tau_{\mathrm{in}}=\frac{1}{\alpha\left(c_{1}+c_{2}\right)} \frac{M_{n}-1}{M_{n}} .
$$

In the case of holes one simply interchanges $\alpha$ and $\beta$ in (49) and (50).

For a statistical analysis we proceed along the same 
lines as in section 3. Applying equation (5) to (48), the following partial differential equation for the GF of the number of pairs is obtained :

$$
\frac{\partial P}{\partial t}=\frac{N_{s n}}{\tau_{\mathrm{in}}}(s-1) P+\frac{1}{M_{n} \tau_{\mathrm{in}}}(1-s) \frac{\partial P}{\partial s} .
$$

In equilibrium :

$$
M_{n} N_{s n} P=\frac{\partial P}{\partial s}
$$

from which :

$$
P=C e^{M_{n} N_{s n} s} .
$$

Since for $s=1, P=1$, we finally obtain :

$$
P=e^{M_{n} N_{s n}(s-1)} .
$$

Transforming back :

$$
p_{n}=\frac{\left(M_{n} N_{s n}\right)^{n} e^{-M_{n} N_{s n}}}{n !} .
$$

This shows that according to

$$
\frac{\partial N}{\partial t}=-\frac{N}{M_{n} \tau_{\mathrm{in}}}+\frac{N_{s n}}{\tau_{\mathrm{in}}},
$$

the spectrum becomes :

$$
S_{N}(f)=4 \overline{\Delta N^{2}} \frac{\tau}{\omega^{2} \tau^{2}+1} .
$$

As in section 3, but now with

$$
\tau=M_{n} \tau_{\text {in }} \text { and } \overline{\Delta N^{2}}=M_{n} N_{s n}
$$

the spectral density is written as :

$$
S_{N}(f)=4 M_{n}^{2} N_{s n} \frac{\tau_{\mathrm{in}}}{\omega^{2} M_{n}^{2} \tau_{\mathrm{in}}^{2}+1} .
$$

As can be seen from (54), there exists no cubic relationship between $S_{N}(f)$ and the multiplication factor $(s)$. The construction of (48) [7] has destroyed some statistical information. Equation (48), as it stands, has a Poissonian character according to equation (51). All sources however, that contribute to the increase or the decrease in the number of pairs should be stated explicitly. Statistically speaking, $(a-b) n$ is not equivalent with $a n-b n$. For a rigorous treatment that can be extended to the nonlinear case, other ways have to be found since the continuity equation approach now fails.

For obtaining the time dependency in the case of unequal ionization coefficients and drift velocities, it has been, and still is, common practice to perturb the system and calculate its frequency dependence and then introduce the frequency dependent parameters into the statistical calculation $[7,11,12]$. A small perturbing electric field of the form

$$
E(t)=E_{0}+\tilde{E} \mathrm{e}^{j \omega t}
$$

is introduced for instance, with which the perturbations in the ionization coefficients and multiplication factors can be calculated. The time dependency of the continuity equation then yields the frequency dependency after introducing these perturbed quantities. It is then shown that by introducing

$$
I=I_{0}+\tilde{I} \mathrm{e}^{j \omega t}
$$

for the current (caused by the electric field perturbation) into the continuity equation, that the multiplication factor can be written as

$$
M(\omega, x)=\frac{M(x)}{j \omega \tau+1}
$$

where in our case $\tau=M_{n} \tau_{\text {in }}$ and $M(x)=M_{n}$. The multiplication factor in the random (shot-noise) source of [10] is then replaced by the one given by (55), which then yields the expressions from [11]. The result for our simplified case (no internal generation, single injection) can be written as $[11,12]$

$$
S_{I}(f)=\frac{2 e I_{s n}}{1+\omega^{2} M_{n}^{2} \tau_{\mathrm{in}}^{2}}\left[M_{n} M_{p}\left(M_{n}-1\right)+M_{n}^{2}\right]
$$

where $M_{n}$ and $M_{p}$ stand for the multiplication factors for electrons and holes respectively. It may be interesting to note that

$$
M_{n} M_{p}\left(M_{n}-1\right)
$$

represents the variance of the probability density function found in [13] and follows also from the implicit equation for the moment GF for the pairs found in [14].

As for the sinusoidal perturbation, two objections arise if the statistical treatment is to be extended to the nonlinear case.

(i) In general it will not be correct to excite a system deterministically in order to obtain its timedependency in the statistical sense. For systems that can be linearized the fluctuations from equilibrium will be small enough sothat the macroscopic equation governing the relaxation back towards equilibrium can be used for the determination of the autocovariance. A sharp peak for the equilibrium density only exists if the system allows a Fokker-Planck description as far as its probability density function is concerned. In other words, only those systems that allow a diffusion limit in the stochastic sense [3] can be perturbed deterministically for obtaining its spectral density. Since the multiplication process is rather noisy, these small deviations from equilibrium almost certainly do not exist in the nonlinear case. It should 
be noted that even in the linear case for small multiplications the equilibrium density becomes highly dispersive [13].

(ii) The response of the avalanche is calculated on the basis of the steady-state continuity equations (19) and (20). These equations do not incorporate the transient. In the linear case this becomes important at high frequencies with a possible influence at intermediate frequencies for strongly differing drift velocities.

In order to avoid these objections, the same problem is discussed in the next section on the basis of the invariant imbedding technique [8 I, $8 \mathrm{II}]$.

5. Reflection statistics for $\alpha \neq \beta$ and $c_{1} \neq c_{2}$. With the invariant imbedding technique a particle is injected and the returning (reflected) particles are studied as a function of time, thus including the transient in the analysis. It should be kept in mind however, that for the nonlinear case equation (40) does not hold either. The only possibility left is a full stochastic treatment on the basis of equation (5). On the other hand, equation (40) takes more accurately the high frequency behaviour and the influence of unequal drift velocities into account [8 I] than the analysis on the basis of the continuity equations (19) and (20).

Our treatment here will be based on an extension of Campbell's theorem ([8 II], see also for further refs.) or the more general result for linear systems [20]

$$
S_{\text {out }}(f)=S_{\text {in }}(f)|G(f)|^{2}
$$

where $G(f)$ represents the system function (Avalanche region).

Since the multiplication is random, the final result will then be the ensemble average (Ergodic hypothesis, section one) :

$$
\overline{S_{\text {out }}(f)}=S_{\text {in }}(f) \overline{|G(f)|^{2}} .
$$

The condition of a linear system implies that the active region must be describable by a linear differential equation as far as the particle number is concerned. The same condition holds if a-c perturbations are used. The second order terms must vanish sufficiently rapidly. It is therefore that equation (5) becomes the only alternative for the nonlinear case.

For a shot-noise input (electrons) with a spectral density $2 e I_{s n}$ the system function $G(f)$ can be established. The integro-differential equation for the rate of emergence (eq. (40)) has the following Laplacetransform [8 I] :

$$
\frac{\partial r^{*}}{\partial x}=\frac{\beta}{c_{2}}-\frac{s}{c_{p}} r^{*}+(\alpha+\beta) r+\alpha c_{1}\left(r^{*}\right)^{2}
$$

where

$$
r^{*}(s, x)=\int_{0}^{\infty} r(x, t) \mathrm{e}^{-s t} \mathrm{~d} t
$$

The solution of this Riccati equation is :

$$
r^{*}-r_{1}=\left(r^{*}-r_{2}\right) C \exp \left[\alpha c_{1} x\left(r_{1}-r_{2}\right)\right]
$$

where $C$ can be obtained from the boundary condition $r^{*}(0, s)=0$ wich yields for $C=r_{1} / r_{2}$ and for which $r_{1}$ and $r_{2}$ are the solutions from :

$$
\alpha c_{p}\left(r^{*}\right)^{2}+\left(\alpha+\beta-\frac{s}{c_{p}}\right) r^{*}+\frac{\beta}{c_{2}}=0 .
$$

It is difficult to find the transform because of the complicated character of (60). On the other hand, as is clearly shown by the convolution, we must be carefull to consider equation (40) as being representative for the mean motion. For small times (not too small for the convolution being noticeable) the process is correlated and the system function cannot be used without difficulty in the statistical analysis. The process is therefore considered at times where the memory character of the convolution can be neglected. For further details see [8 I]. It was found for the spectral density ([8 I $]$ equation (12)) :

$$
S_{I}(f)=\frac{2 e I_{s n}}{\omega^{2} \tau^{2}+1}\left[M_{p} M_{e}\left(M_{p}-1\right)+\left(M_{p}-1\right)^{2}\right]
$$

where for unequal ionization coefficients (of the same order) :

$$
\tau=\frac{M_{p}-1}{2 c_{p}}, \quad c_{p}=\frac{c_{1} c_{2}}{c_{1}+c_{2}} .
$$

Equation (62) agrees with the standard results [11, 12] apart from $\left(M_{p}-1\right)^{2}$ instead of $M_{p}^{2}$, since it is dealt with the returning particles (the injected one is missing).

The advantage of the invariant imbedding technique for this two point boundary value problem is not only the fact that it is started with first principles from probability theory without using such tricks as the Langevin source or an a-c perturbation, but also the way it can handle the transient and the evaluation of the multiplication factor and its statistics for unequal drift velocities. For a discussion about the drift ' velocities in the intrinsic time constant on the basis of the steady-state continuity equations (19) and (20) and the time constant as is given by (63) we refer to [8 I].

6. Nonlinear statistics. - The foregoing analyses showed that it is possible to recover the bulk of the statistical results from the ideal avalanche diode starting from first principles in the statistical sense and therefore unifying the various treatments in such a way that the nonlinear case can be included. This is not an easy task. To our knowledge, the simplest inherent nonlinear problem has hot been solved yet rigorously in the statistical sense. The problem is 
usually linearized (G.-R. theorem) or treated in the thermodynamic limit. One uses tacitly the large system assumption, that is, the powerfull property that the majority of the physical systems can be described by the Fokker-Planck approximation. To a certain extent, this even holds for the master equation expansion which is more accurate however than the usual diffusion limit. Other expansions have been proposed and different moment schemes presented for the infinite hierarchy of differential equations that usually arise for nonlinear phenomena. We shall not go into this any further.

Apart from these theoretical complications there is also the difficulty to find a suitable differential equation for the pairs in the active region on the basis of such nonlinear effects as space charge. A first attempt has been made in [21]. The number of ionizing events per transit were taken to be a function of the number of created pairs. Langevin methods and a diffusion approximation (Burgess' method) were applied to obtain the probability density function of the equilibrium number density of created pairs. These procedures are equivalent to those from $[7,9$. $10,11,12]$ for the stationary and time dependent fluctuation properties of the ideal diode. We shall show now by means of a simple example that these methods may become inadequate for a stochastic treatment of a nonlinear problem.

Suppose that the avalanche is self-sustaining with an injection $n_{s}$ and that the continuity equation for the evaluation of the number of pairs with respect to time can be written as :

$$
\tau_{t} \frac{\mathrm{d} n}{\mathrm{~d} t}=\gamma(n)-n
$$

The Read equation with a number density dependent ionization coefficient could serve as a model for (64). We now assume an exponential dependency

$$
\gamma(n)=\mathrm{e}^{-q n} n+n_{s}
$$

taking into account the decrease in ionizing events with the increase of the number of pairs in the active region (space charge influence for instance). For a weak interaction, equation (64) is written as :

$$
\tau_{t} \frac{\mathrm{d} n}{\mathrm{~d} t}=n_{s}+(1-q n) n-n .
$$

This yields a mean value (deterministic, not stochastic !)

$$
n_{0}=\sqrt{\frac{n_{s}}{q}} .
$$

It is now assumed that the diffusion approximations from $[7,9,10,11,12,15]$ still hold (Langevin, Burgess' method, perturbations, etc.). This implies that the fluctuating phenomenon (number of pairs in the active region) is replaced by an Ornstein-Uhlenbeck process [5]. Its time constant can be obtained from equation (66) (Burgess' method for instance [15]) :

$$
\tau^{-1}=2 q n_{0} \tau_{t}^{-1} \text {. }
$$

The variance is equal to half the product of the socalled infinitesimal variance (= spectral density of the white source in the Langevin sense) [3] and the time constant :

$$
\overline{\Delta n^{2}}=1 / 2 \sigma^{2} \tau=n_{0} \tau_{t}^{-1} \cdot\left(2 q n_{0}\right)^{-1} \tau_{t}
$$

or

$$
\overline{\Delta n^{2}}=\frac{1}{2 q} .
$$

For the exact statistics in equilibrium, the following relationship has to be satisfied :

$$
\overline{n^{2}}=\frac{n_{s}}{q}
$$

as can be seen from equation (66). The problem is now to find the mean of the process. Constructing a diffusion limit such as Burgess' method, the G.-R. theorem or the Langevin approach, implies that the deterministic mean given by (67) is to be equivalent with the stochastic mean (sharply peaked equilibrium density). For a more general discussion, see [5]. Applying this, results in

$$
\overline{\Delta n^{2}}=\overline{n^{2}}-n_{0}^{2}=0
$$

as can be seen from (70) and (67). This is not in accord with (69). Even more convincing is the comparison between equations (69) and (70) where the former represents the usual approximative result and where equation (70) is exact. Particularly for a small injection, $\overline{n^{2}}$ can almost be identified with $\overline{\Delta n^{2}}\left(=\overline{n^{2}}-\overline{n^{2}}\right)$. In other words, the stochastic mean is almost equal to zero. Since for a realistic photodiode the number of pairs in the active region easily attains values of orders of magnitude, other ways than the classical ones have to be found if the noise analysis of an ideal photodiode is to be extended to the nonlinear regime.

7. Discussion. - Even for the ideal diode, a statistical analysis of the multiplicative phenomena is a complicated matter. For engineering purposes the main problem is to find a spectral density and probability density function for the number of pairs in the active region. Any theoretical analysis can be divided into two categories.

(i) It is started with the continuity equations in the steady state;

(ii) A carrier is injected and the emerging current is investigated.

In the first case, one assumes a stationary and linear (or even ergodic) process. Once the macroscopic differential equation is known, the spectral density can be calculated by means of equation (5), without using 
random sources, a-c perturbations, etc. This equation can be considered as a common basis for the various treatments so far. Accepting this picture, it then seems to be legitimate to use the continuity equations (19) and (20) for this purpose. The actual process is of a more complicated nature however. For an injection rate that is large compared with the reciprocal time constant of the multiplication process the abovementioned coarse-grained procedure probably works. In practical diodes the reciprocal generation rate is much larger than the relevant time constant. For frequencies well below $\tau_{t}^{-1}$, the noise will be white and the signal can be represented by a series of sharp pulses with varying amplitude. At high frequencies the shape of the pulses introduces a frequency depression factor which is not adequately described by those treatments based upon the continuity equations $[7,9,10,11,12]$.

In the second case, the nonstationarity of the multiplied current is taken into account. In the high frequency regime and for unequal drift velocities, the intrinsic response time then shows deviations. In practice, the central idea of a multiplied transittime or time between ionizations appears to be fruitfull however. We nevertheless assume, that on those occasions where the intrinsic response time concept has been verified experimentally, the saturated driftvelocities were of the same order of magnitude. Discrepancies arise otherwise with the stationary response time and the nonstationary ones from the invariant imbedding technique. (For a more complete discussion, see [8 I] section 4.2.) In order to show this, a step function is applied to the diode and the current transient is studied. It was found in [7] on the basis of the continuity equations :

$$
I(t)=M_{n}\left[1-\frac{M_{n}-1}{M_{n}} \mathrm{e}^{-t / \tau}\right]
$$

with

$$
\tau=M_{n} \tau_{\text {in }}=\frac{M_{n}-1}{\alpha\left(c_{1}+c_{2}\right)}
$$

where we have used a unit input current and where $M_{n}$ is the multiplication factor for electrons and $\tau_{\text {in }}$ the intrinsic response time. This is how the system approaches steady state after a heaviside perturbation of the electric field for instance. The invariant imbedding technique showed a more complete result which only could be approximated by using a Tauberian theorem [8 I] (by means of which the initial stages of the transient were suppressed) for the evaluation of the total number of emerging particles, with respect to time and after a single injection, it was found :

with

$$
R(t)=2\left(M_{n}-1\right)\left[1-\mathrm{e}^{-t / \tau}\right]
$$

$$
\tau=\frac{M_{n}-1}{\alpha c_{p}}, \quad c_{p}=\frac{c_{1} c_{2}}{c_{1}+c_{2}}
$$

For a multiplication which is large enough both results seem to be in agreement if one neglects a factor of two. The time constants are different however. If $c_{1} \neq c_{2}$, for instance, the time dependency should be governed by the smallest drift velocity. This is the case for (73) but not for the intrinsic response time.

It may seem at first sight that for $\alpha \neq \beta$ and $c_{1} \neq c_{2}$ it cannot be started with equation (5) since the functional rates of increase and decrease of the avalanche process are not well described by the differential equation (48) which is found by combining (19) and (20) [7]. This combination destroyed some statistical information. We therefore used some results from the invariant imbedding technique, which led to equation (62). Since equation (5) is of a universal validity there should be a link between both procedures.

To that purpose the following points may be relevant :

1) Any noise analysis of a linear problem or one that can be linearized (diffusion limit) can be handled with equation (5) as soon as the functional rates of increase and decrease are known. The time independent fluctuations can be obtained from the partial differential equation of the probability transform and the time dependent properties from the macroscopic continuity equation.

2) The majority of the statistical problems in physics are well described by a macroscopic equation with microscopic rate processes that are additive (independent increments). This means that the statistics of many problems can be described by equation (5), with rates of increase and decrease that can be considered as shot noise sources.

In order to illustrate this connection between equation (57) and equation (5), it is supposed that the macroscopic equation can be written as :

$$
\frac{\mathrm{d} n}{\mathrm{~d} t}=a-b n .
$$

Note its resemblance with equation (48) for $\alpha \neq \beta$ and $c_{1} \neq c_{2}$. The spectrum is written as (see section 3 for details) :

$$
S_{n}(f)=4 \overline{\Delta n^{2}} \frac{\tau}{\omega^{2} \tau^{2}+1}, \quad \tau=\frac{1}{b} .
$$

Using equation (5), the variance is found to be $a \tau$. On the average the system is excited by a shot noise source $2 a$ (increase plus decrease). Using :

$$
e^{2} S_{n}(f)=S_{I}(f)
$$

where $e$ is the electronic charge, the current spectral density becomes :

$$
S_{I}(f)=2 e I \frac{\tau^{2}}{\omega^{2} \tau^{2}+1}=2 e I|G(f)|^{2}
$$

where $G(f)$ represents the system function $(j \omega+b)^{-1}$. 
Concluding it can be said, that, since the classic avalanche treatments may fail (section 6) in the nonlinear case, possible candidats for a statistical analysis are the following :

I) The process in studied by means of a continuity equation, which now becomes nonlinear (eq. (66) for instance). A complete analysis is then possible on the basis of equation (5). The encountered difficulties are :

(i) The construction of a macroscopic equation that takes the physical processes adequately into account.

(ii) The solution of the arising partial differential equation for the probability transform. One easily encounters orthogonal polynomials and special functions in general. An infinite hierarchy of moment equations has to be solved.

(iii) The macroscopic continuity equations describe only the average behaviour of the process and are inadequate to describe the pulse behaviour.

II) The process is studied by means of the invariant imbedding technique. The encountered difficulties are :

(i) The spatial dependency of the ionization coefficients needs to be calculated. (ii) For the system function, implicit equations are expected because of the nature of this two point boundary value problem.

8. Conclusion. - In order to extend the noise analysis of an ideal avalanching diode to the nonlinear regime (space charge effects) it has been tried to relate the relevant results from avalanche noise theory to a general equation for Markov Processes, which enables a nonlinear treatment. It could be shown that :

(i) The Read equation can be solved rigorously in the statistical sense (including the time dependency of the moments).

(ii) The reflection statistics and the invariant imbedding technique take the pulsed character of the Avalanche better into account than those methods based on the continuity equations.

(iii) Avalanche noise theory extensively deals with linear methods that may fail for nonlinear phenomena.

(iv) All known results in the time independent case follow from a simple multiplicative process with a birth parameter equal to $\beta-\alpha$.

\section{References}

[1] Bartlett, M. S., An Introduction to Stochastic Processes (Cambridge University Press) 1955.

[2] Moyal, J. E., Roy, J., Stat. Soc. 2 (1949) 150.

[3] Cox, D. R., Miller, H. D., The Theory of Stochastic Processes (Chapman and Hall) 1965.

[4] FelleR, W., An Introduction to Probability Theory and its Applications, I, II (John Wiley) 1971.

[5] Walma, A. A., Noise in Physical Systems, Ed. D. Wolf (Springer Verlag) 1978.

[6] Read, W. T., Bell Syst. Tech. J. 37 (1958) 401.

[7] LeE, C. A. et al., J. Appl. Phys. 38 (1967) 2787.

[8] Walma, A. A., I Solid State Electron. 18 (1975) 511. II Solid State Electron. 19 (1976) 1. III Solid State Electron. 19 (1976) 167.

[9] TAGER, A. S., Sov. Phys. Solid State 6 (1965) 1919.

[10] MCINTYRE, R. J., IEEE Trans. ED-13 (1966) 164.
[11] NaGvi, I. M., Solid State Electron. 16 (1973) 19.

[12] Ringo, J. A., Lauritzen, P. O., Solid State Electron. 15 (1972) 625.

[13] MCINTYRe, R. J., IEEE Trans. ED-19 (1972) 703.

[14] Personick, S. D., Bell. Syst. Techn. J. 50 (1971) 3075.

[15] Vliet, K. M. V., FAsset, J. R., In : Fluctuation Phenomena in Solids. Ed. R. E. Burgess (Ac. Press) 1965.

[16] Balaban, P., Fleischer, P. E., Zucker, H., IEEE Trans. ED (1976) 1189.

[17] Emmons, R. B., J. Appl. Phys. 38 (1967) 3705.

[18] MCKelveY, J. P., Solid State and Semiconductor physics (Harper Row) 1966.

[19] Kuvas, R., Lee, C. A., J. Appl. Phys. 41 (1970) 1743.

[20] Papoulis, A., Probability, Random variables and Stochastic Processes (Mc Graw-Hill) 1965.

[21] Aladinski, V. K., Sov. Phys. Semicond. 6 (1973) 1731. 